\title{
Facial Skin Smoothening by Iterative Steering Kernel Regression Technique
}

\author{
Ms. Priyanka Mehra ${ }^{1}$, Prof. A. S. Deshpande ${ }^{1}$ \\ Imperial College of Engineering and Research, Wagholi, Pune ${ }^{1}$
}

\begin{abstract}
The paper enhanced the facial skin using iterative steering kernel regression which is based on edge preserving energy minimization. The region-aware mask allows the user to beautify faces with three major skins attributes homogeneity, lighting, and color. The main aim of this paper is to beautify the skin by decomposition into the three layers using edge preserving filter. After layer decomposition a facial landmarks and significant features extracted from the database to generate the mask into three different layers which allow the user to perform facial beautification simply by adjusting its skin parameter. The combination of these parameters can be optimized automatically it depend on the data base or knowledge base. The experiment result shows that the image is enhanced more as compare to the original image. After three region-aware masks have been obtained, a user can perform facial beautification simply by adjusting the skin parameters. Result of proposed method will be compared with the existing methods in terms of Peak Signal to noise ratio (PSNR) and Mean Square Error (MSE).
\end{abstract}

Keywords: Feature Extraction, Facial Beautification, Edge Preserving Energy Minimization, Adaptive Edge Preserving Energy Minimization, Iterative steering Kernel Regression.

\section{INTRODUCTION}

Facial skin smoothing is a widely used technique for advertisement, magazines, and websites which manipulate facial image every day. Some commercial systems are available (such as Adobe Photoshop) but it is a timeconsuming task. Moreover, image enhancement and image distribution are becoming more popular as social networks (e.g., Facebook, LinkedIn, and Flickr). According to current research, the facial skin smoothening generate mask into two ways. Facial mask generated a specific image segmentation problem; the segmentation model [1][3] contains the facial priors (such as the skin color or shape) which integrate into layer.

This approach avoids the tedious task of mask painting, but the mask boundaries often fail to follow the region boundaries closely, which may introduce visual artifacts. Another method for improving mask generation is to construct a much simpler and more interactive manipulation tool. The method of edit propagation technique propagates scarce user edits all over the entire image according to the pixel affinity. A significant advantage of edit propagation is its edge-aware property, which produces adaptive transitions between the boundaries of different regions without any user intervention. During facial skin enhancement, various regions need to have different edge-aware levels, depending on their editing properties.

However, homogeneous parameters of existing edit propagation may fail to produce specific variable diffusion effects for facial masks, while interactive initialization appears to be cumbersome for beautification tasks. The facial skin appearance and color are important attractive factors, and mostly people consider that a good picture should be blemish free and have smooth facial colors. The proposed method of an automatic region aware maskovercome the limitations of these method for facial skin smoothening, by using edge preserving energy minimization framework introduced by Lischiniski et al. [4].

Leyvand et al. [5] proposed the "novel computational photography technique" to enhance the visual appeal of human face images while keeping high similarity with the original image [5], [1].The Photographytechnique can be used for Advertisement, Magazines, website manipulation.Chen et.al [6] proposed "skin color detection" algorithms to extract the skin parts from image. Mostly the numerical method used to find out the boundary of human skin color in particular color space. For preprocessing step to find out the minor effect of color temperature which is better for us then, user can find out the characteristic of skin color. Farbman et al. [7] proposed edit propagation technique it is work on "Diffusion maps for edge- aware image editing". By using this technique it edits throughout the entire image according to the pixel affinity also it works on manifold preserving edit propagation method.

C. florea [8] proposed "skin beautification algorithm" consists of the following steps. Face detection and tracking, Skin detection, Skin enhancement. Peer et.al [9] proposed suitable solution is the set of conditions. A pixel is being described in terms of its $\mathrm{R}, \mathrm{G}$, and $\mathrm{B}$ components. For the second stage use thresholding on the PCA principal axes derived from skin color . This technique has 
increased computational burden so processing is distributed over two or more consecutive video frames.D.GUO [10] proposed the "digital face make algorithm" is inspired by the process of physical makeup. The decomposition of the subject and example images into three layers separately: face structure Layer, skin detail layer, and color layer.

Ideally, the face structure layer contains only the structure of every face component such as the eyes, nose, mouth, etc. Huang et al. [11] constructed a challenging face segmentation data set" from the labeled face in the wild (LFW) database, and they trained a standard conditional random field (CRF) model [12] to build a hair/skin/background labeler. Wang et al. [13] proposed a "compositional exemplar-based model" that regularizes the output of a segmentation using parts Scheffler et al. [14] trained a labeler that combines color models, special prior, and a Markov random field model. Kae et al. [15] integrated the global shape prior into CRF to construct the GLOC (Global and Local) model for skin segmentation. However, directly using this model may introduce visual artifacts for skin beautification because (super) pixel-wise skin labeler produces mask with rigid boundary. Scherbaum et al. [2] implemented a computer based 3-D makeup-mapping program that used facial examples with and without professional makeup. Tong et al. [16] generated cosmetic makeup by transferring the appearance properties of a face with makeup onto a novel face image, Guo and Sim [10] decomposed input and example faces into detail, structure, and color layers, before digital makeup was implemented by blending the corresponding layers of the two faces.

\section{FACIAL SKIN SMOOTHENING}

Facial skin smoothening based on adaptive edge preserving energy minimization technique which is used to enhanced image by using adaptive region aware mask method. Block diagram of facial skin smoothening is as given in below figure 1 .

Facial skin smoothening based on edge preserving energy minimization method which is decomposed into three different layers such as lightning, smoothness, color. After decomposition, landmarks are located for feature extraction of several region of layer. After decomposition masks is generated for different layer which beautify the image. The input image is enhanced by using iterative steering enhancement (kernel regression) technique. Which worked on iterative steering algorithm as well as Q meter is used to identify the anisotropic patches and removes some artifacts on the image.

The steps in beautification process:

1. The step for execution is to take input image from the data base.

2. After taking the input image from the folder where several images are stored, face is extracted.
3. The image layer decomposition method has three different layers such as color intensity, compression, Intensity of layer.

4. The use of edge detection create the landmarks or called as control points of different layer .For different layer of region different masks is generate.

5. Different layer of image is extracted by image layer decomposition which genearte masks for different region of layer such as lighting, smoothness,color layer.

6. The detail layer of features extracted from the image of input is in the form of numerical data. The detail layer of frequency called as low frequency data and high frequency data.

7. Traditional method is used to enhance the facial skin.

8. Proposed method is used to enhance the input image more clearly as compare to traditional method by using parameters.

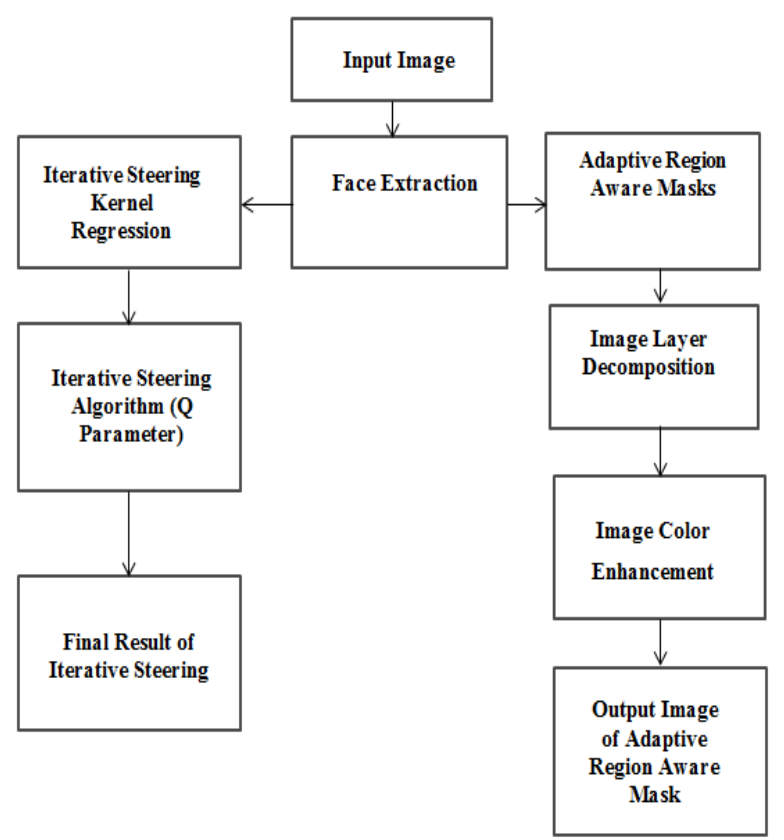

Fig.1 Block Diagram of Facial Skin Smoothening

\section{A. Image Layer Decomposition}

Image layer decomposition of framework decomposed into skin lighting, homogeneity, and color. Image is detached into three image layers according to the skin attributes, as shown in fig.2. In CIELAB color space input image $\mathrm{I}$ is converted which is commonly used for facial attractiveness [17]-[19].

The converted input image is composed into two layer one is lightness layer $\mathrm{L}^{*}\left(\mathrm{I}_{\mathrm{L}}{ }^{*}\right)$ and two color layer $\mathrm{a}^{*}\left(\mathrm{I}_{\mathrm{a}}{ }^{*}\right)$ and $\mathrm{b}^{*}\left(\mathrm{I}_{\mathrm{b}}{ }^{*}\right)$. The chromaticity channel (color) are regarded as color layer $\mathrm{I}_{\mathrm{C}}$. Next the smoothing filter is applied to the (lightness) layer to capture its large-scale variations, which is viewed as the lighting layer $\mathrm{I}_{\mathrm{L}}$. Last, the lighting layer is subtracted from $\mathrm{I}_{\mathrm{L}}{ }^{*}$ and the result is obtained as the detail layer $\mathrm{I}_{\mathrm{S}}$. 
IJIREEICE

ISO 3297:2007 Certified

Vol. 5, Issue 1, January 2017

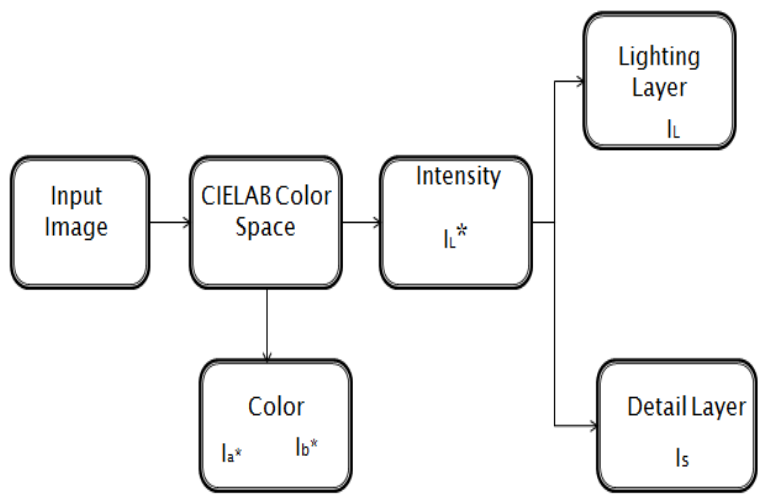

Fig.2 Process to Separate the Input Image into Facial Skin Layer

\section{$\mathbf{I}_{\mathbf{L}}{ }^{*}=$ Intensity, $\mathbf{I}_{\mathbf{S}}=$ Detail Layer, $\mathbf{I}_{\mathbf{C}}=$ Color Layer}

The image layer decomposition decompose different layer of image such as lightining, smoothness, color. Decomposition of image from the input is converted into color space called as "CIELAB" where it can decompose into lightness and color layer,after applying the smoothing filter or called as gradient,lightining layer is formed ,this lightning layer when it subtract from the lightness layer then smoothness layer is formed.

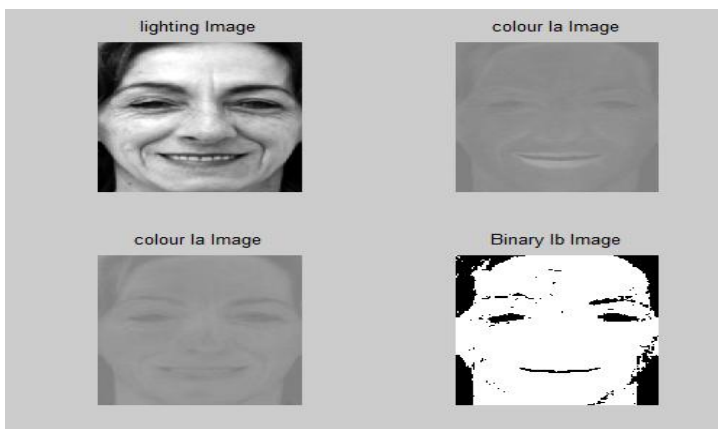

Fig.3 Different Layers of Facial Skin Smoothening

\section{B. Feature Extraction}

For facial skin smoothening the main aim of feature extraction is to find out the landmarks for mask generation. The important features of facial components such as (chin, lips, and nose). All this important feature detectors integrate into the framework, but this implementation is not an easy task. The important features analyzed by Viola-Jones face detector [20] and (ASM) [21].

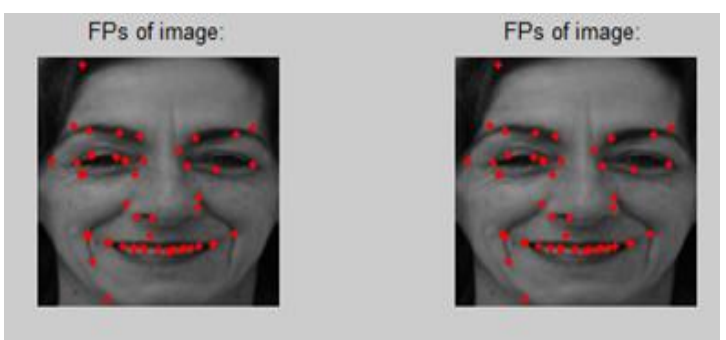

Fig.4 Features points
In facial skin smoothening figure represent the features extraction. It is nothing it takes the detail from the image, by using the DCT for the feature extraction, extracted features from the image, the extracted features in the form of numerical.

\section{ADAPTIVE REGION AWARE MASK}

The adaptive region aware mask generation is used to remove unwanted details such as (spots), wrinkles or manipulate the skin color or it adjust facial qualities (such as the skin color) in certain face skin regions, without disturbing other information in such regions like background, simultaneously modified region control the degree of adjustment using specific layer masks for skin lighting, smoothness, and color enhancement.

In our method, the extract facial features are treated as input constraints. The guarded regions are propagated pixel values flexibly all over the entire image according to the guided information. By using edge preserving energy minimization method generate region aware mask generated which was originally proposed by Lischiniski et al. [4] for tonal adjustment.

By using edge detection database implementation on two methods, first the face extracted from the image of input stored in the database folder then simultaneously feature is extracted and layer decomposition such as, lightning, color, smoothness, for this three layer mask is generated by using region aware mask, after generating mask image layer is manipulated by interactive and automatic layer enhancement.

$$
\begin{aligned}
& M=\operatorname{argmin}_{M}\left\{\sum_{z} w(z)(M(z)-R(z))^{2} \sum_{Z} h(\nabla M, \nabla G)\right\} \\
& h(\nabla M, \nabla G)=\lambda\left(\frac{\left\|M_{X}\right\|^{2}}{\left\|G_{X}\right\|_{P}^{\alpha(z)}+\varepsilon}+\frac{\left\|M_{Y}\right\|^{2}}{\left\|G_{Y}\right\|_{P}^{\alpha(z)}+\varepsilon}\right)
\end{aligned}
$$

The first term in (1) is the data term, which ensures that mask $M$ satisfies the facial constraint $R$. The weight $w$ is used to represent the constrained pixel, where $w \in[0,1]$. A larger weight value indicates that the values of $M$ and $R$ will be more similar.

The second term in equation (1) is the smoothing term, which is responsible for keeping the gradients of the mask $\mathrm{M}$ as low as possible, the main factor represent the $G$ called as gradient. We generalize the guided image from the original model of Lischiniski et al. [4] to a guided feature space, which means that richer facial information can be used to guide the propagation. Three parameters control propagation in the smoothing term: $\varepsilon$ is a small constant that prevents division by zero, $\lambda$ is used to balance the relative weights of the two terms, and $\alpha$ controls the propagation sensitivity to the gradients of $\mathrm{G}$.

\section{LAYER MASKS}

After feature extraction from the image as well as layer decomposition for smoothing different layer of mask is generate such as lighting mask, smoothness mask, and color mask. 
Lighting Mask:

The intensity of the mask indicates the edit degree of the process, where brighter region will be adjusted more.

Smoothness Mask:

During skin smoothness enhancement, we need to handle some highly complex situations. For example, unwanted textures such as wrinkles or spots need to be removed whereas significant facial information must be preserved, such as details of the eyes, hair, or beards. To generate the smoothness mask, the guided feature is constructed using the facial lighting and the color feature as follows:

$$
\mathrm{G}=\mathrm{G}_{\mathrm{s}}=\left(\mathrm{I}_{\mathrm{L}} *, \mathrm{I}_{\mathrm{a}}{ }^{*}, \mathrm{I}_{\mathrm{b}} *\right)
$$

Where $\mathrm{I}_{\mathrm{L}}{ }^{*}, \mathrm{I}_{\mathrm{a}}{ }^{*}$ and $\mathrm{I}_{\mathrm{b}}{ }^{*}$ are the normalized luminance and chromaticity channels of the input image in CIELAB space and guided feature $\mathrm{G}_{\mathrm{s}}$

\section{Color Mask:}

The process used to generate the color mask is similar to that employed to produce the smoothness mask. The deatil layer of features extracted from the image of input is in the form of numerical data.The detail layer of frequency called as low frequency data and high frequency data.The edge preserving energy minimization of low frequency data is preserved and high frequency analyzed low data.

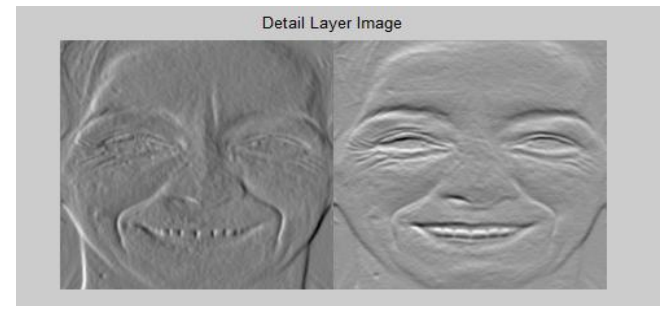

Fig.5 Detail Layer

E. Edge Detection

The use of Edge detection create the landmarks or called as control points of different layer .For different layer of region different masks is generate. The control points of edges shows the features point where the implementation is done by using canny edge detector algorithm.

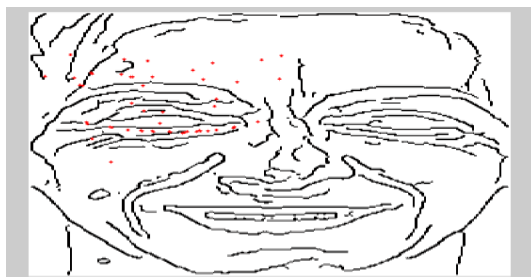

Fig.6 Control points of Edge

\section{ITERATIVE STEERING KERNEL REGRESSION}

The expected smoothing matrices of the steering kernel regression method are data dependent, and, subsequently, delicate to the noise in the input image. when an iterative denoising procedure is used to deed the output (less noisy) image of each iteration to evaluate the radiometric terms of the kernel in the next iteration.

A block diagram representation of this method is shown in below figure, where $\mu$ is the iteration number. In this diagram, the initial gradient of estimation of data samples creates the initial data where noisy image is found out by iteration method.
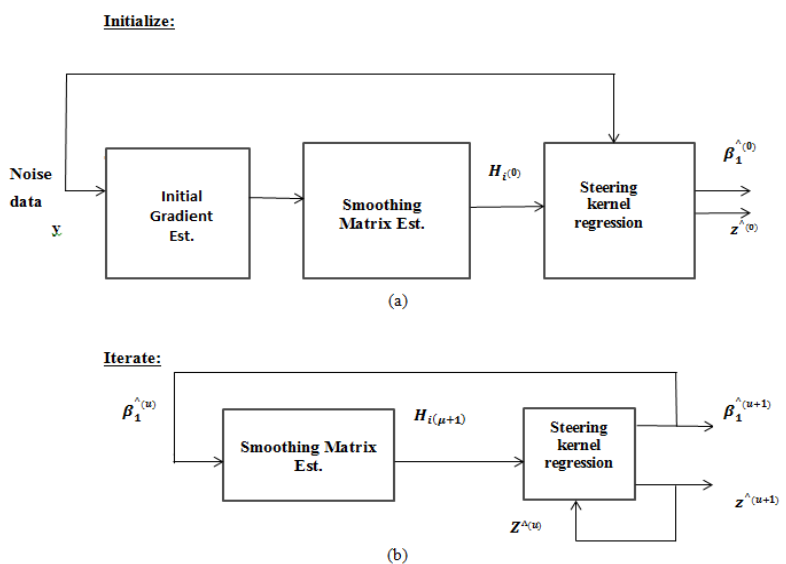

Fig.7 Iterative Steering Kernel Regression

In the following iteration, the image is reconstructed which is used to analyze a more dependable estimation of the gradient fig.7 (b) and this process continues for a few more iterations. In Fig 7(a) shows that, proposed algorithm depend on an original estimation of the gradient, we directly apply the estimated kernels on the original (non interpolated) samples which results in the populated (or denoised) image of first iteration .Therefore, denoising and interpolation are done jointly in one step. Further iterations in Fig.7 (b) apply the modified kernels on the denoised pixels which results in more aggressive noise removal.

Classical method of kernel regression of image processing rely on a exact model of the signal of interest and try to find the parameters of this model in the presence of noise. There are some example of this approach which is presented in diverse problems ranging from denoising to up-scaling and interpolation.

$$
y i=Z(x i)+\in i
$$

$\boldsymbol{y} \boldsymbol{i}$ - A sample

$\boldsymbol{Z}\left(\boldsymbol{x}_{\boldsymbol{i}}\right)$-The regression function

$(x \boldsymbol{i})$-The sampling position

\section{$\in \boldsymbol{i}$-Zero mean}

where the coordinates of the measured data " $y_{i}$ " is now the $2 \times 1$ vector. Correspondingly, the local expansion of the regression function is given by

$$
\begin{gathered}
Z\left(X_{i}\right)=Z(X)+\{\nabla Z(X)\}^{T}\left(X_{i}-\mathrm{X}\right) \\
+\frac{1}{2}\left(X_{i}-X\right)^{T}\left\{\mathcal{H}_{Z}(X)\right\}\left(X_{i}-X\right)+\cdots \\
=\mathrm{Z}(\mathrm{X})+\{\boldsymbol{\nabla Z}(\mathrm{X})\}^{T}\left(X_{i}-X\right) \\
+\frac{1}{2} \operatorname{vec}^{T}\left\{\mathcal{H}_{Z}(X)\right\} \operatorname{vec}\left\{\left(X_{i}-X\right)\left(X_{i}-X\right)^{T}\right\}+\cdots
\end{gathered}
$$


Where $\nabla$ and $H$ are the gradient $(2 \times 1)$ and Hessian $(2 \times$ 2 ) operators, respectively, and $\operatorname{vec}(\bullet)$ is the vectorization operator, which lexicographically orders a matrix into a vector.

After simplification of above equation (4).

$$
\begin{gathered}
Z\left(X_{i}\right)=\beta_{0}+\beta_{1}^{T}\left(X_{i}-X\right)+\beta_{2}^{T} \operatorname{Vech}\left\{( X _ { i } - X ) \left(X_{i}-\right.\right. \\
X) T+\ldots \ldots \ldots
\end{gathered}
$$

A comparison of (4) and (5) equation

$$
\begin{aligned}
& \beta_{1}=\nabla \mathrm{z}(\mathrm{x})=\left[\frac{\partial \mathrm{z}(\mathrm{x})}{\partial \mathrm{x}_{1}}, \frac{\partial \mathrm{z}(\mathrm{x})}{\partial \mathrm{x}_{2}}\right]^{\mathrm{T}} \\
& \beta_{2}=\frac{1}{2}\left[\frac{\partial^{2} \mathrm{Z}(\mathrm{x})}{\partial \mathrm{x} 1^{2}}, 2 \frac{\partial^{2} \mathrm{z}(\mathrm{x})}{\partial_{\mathrm{x} 1} \partial_{\mathrm{x} 2}}, \frac{\partial^{2} \mathrm{z}(\mathrm{x})}{\partial \mathrm{x}_{2}}\right]^{\mathrm{T}} \\
& \mathrm{K}(\mathrm{Z})=\frac{1}{\operatorname{det}(\mathrm{H})} \mathrm{K}\left(\mathrm{H}^{-1} \mathrm{Z}\right) \\
& \mathrm{H}
\end{aligned}
$$

Where $\mathrm{K}$ is the 2-D realization of the kernel function, and $\mathrm{H}$ is the $2 \times 2$ smoothing matrix.

\section{A. Smoothening}

Usually images which are captured, stored and transmitted over a network might be corrupted by particular method of external noise. Before extracting the edge point, edge detection is important to filter of external noise from the image before extraction. The energy of a typical image is primarily focused in its low-frequency components [22]. This is due to high spatial connection among neighboring pixels. The energy of a wideband chance noise is typically more spread out over the frequency domain. By decreasing the high frequency apparatuses while preserving the lowfrequency components, low pass filtering reduces a large amount of noise while smoothing out the image signal. This in turn reduces the noise in the image. Similar functionality can be achieved by using a Gaussian low pass filter.

$$
h(x)=\frac{1}{\sqrt{2 \pi \sigma^{2}}} e^{-\frac{x^{2}}{2 \sigma^{2}}}
$$

Here $\sigma$ represents the standard deviation of the Gaussian distribution. For applying Gaussian function on images we need a two dimensional function which can be obtained by the product of two one dimensional Gaussian functions.

$$
\left.h(x, y)=\frac{1}{2 \pi \sigma^{2}} e^{-\left(x^{2}+y^{2}\right.}\right) / 2 \sigma^{2}
$$

Where $\mathrm{x}$ and $\mathrm{y}$ represents the pixels along horizontal and vertical directions. The center point of Gaussian distribution with concentric circles of two dimensions, this function produces a surface. The convolution kernel is computed by sampling the values from this Gaussian distribution. Then this convolution kernel is applied on the input grey scale image.

\section{B. Steering Kernel}

In this method local gradient estimation measured between the neighboring pixels value by using this estimation .As an example, if a pixel value is located near an edge, then pixels on the same side of the edge will have much stronger weight with this perception in mind, here it proposed into two steps. Approach of these two steps where first an initial estimate of the image gradients is made using some kind of gradient estimator (say the second order classic kernel regression method).Next step is used to measure the main orientation of the local gradients in the image [23].

In a next filtering stage, this orientation information is then used to adaptively "steer" the local kernel, resulting in elongated, elliptical contours spread along the directions of the local edge structure. With these locally adapted kernels, the denoising is effected most strongly along the edges, rather than across them, resulting in strong preservation of details in the final output. To be more specific, the data-adapted kernel takes the form.

$$
\mathrm{K}_{\text {adapt }\left(\mathrm{x}_{\mathrm{i}}-\mathrm{x}, \mathrm{y}_{\mathrm{i}}-\mathrm{y}\right)} \equiv \mathrm{k}_{\mathrm{H}_{\text {isteer }}\left(\mathrm{x}_{\mathrm{i}}-\mathrm{x}\right)}
$$

Where $\mathrm{H}_{\text {isteer }}$ are now the data-dependent full matrices which we call steering matrices.

\section{EXPERIMENTAL RESULTS}

The facial skin smoothening system is implemented using an adaptive region aware mask technique. The adaptive region aware mask implemented on several original input portraits, it can be observed that by using edit propagation technique the spatially different parameter generates the different layer of masks. The input image of facial skin smoothening is enhanced by using iterative steering kernel regression which remove artifacts, blurriness, etc. The experimental evaluation for our method was worked on face database that is, called as FEI database. Data base covers frontal faces with different background, lightings, expressions, and pose .In experimental result testing faces were eliminated with extreme lighting, expression, and pose variances in the experiments.GUI window contain three images, input image of GUI, by using adaptive region aware mask output image of GUI is implemented and the last image is called as enhanced image which is implemented by applying steering enhancement on facial skin enhancement of input image it work on iterative algorithm and Q meter .

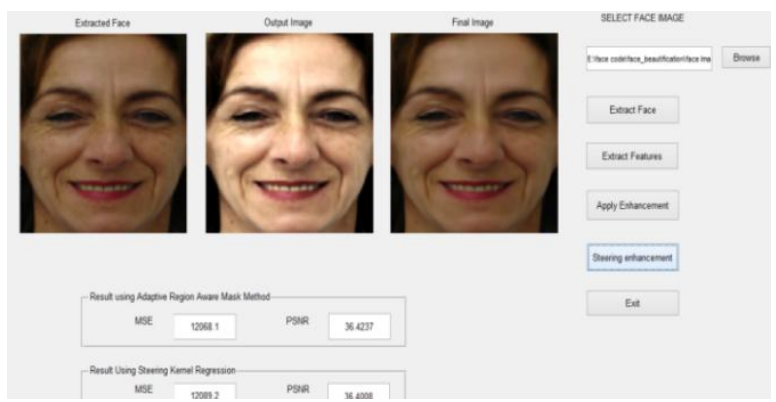

Fig .8 Result of steering kernel regression 
Vol. 5, Issue 1, January 2017

Result of database implementation on two methods, first the face extracted from the image of input stored in the database folder then simultaneously feature is extracted and layer decomposition such as, lightning, color, smoothness, for this three layer mask is generated by using region aware mask. The input image is again enhanced by proposed method called as iterative steering enhancement which removes the noise, blurriness, and artifacts clearly as compare to traditional method.

Calculation of Comparison Parameters PSNR \& MSE:

To determine the visual quality of image, calculate the (PSNR .It can be calculated easily. PSNR can be calculated as,

$$
\text { PSNR }=10 \times \log _{10}^{\frac{\left(2^{\mathrm{K}}-1\right)^{2}}{\mathrm{MSE}}}
$$

Where, $\mathrm{K}$ is no. of bits required representing one pixel, MSE, PSNR can be written mathematically as;

$$
\text { PSNR }=10 \times \log \left(\frac{255^{2}}{\mathrm{MSE}}\right)
$$

Calculation of MSE....

MSE $=\frac{\left.\text { sum (sum }\left((\text { Input Image }- \text { Reconstructed } \quad \text { Image })^{2}\right)\right)}{\mathrm{M} * \mathrm{~N}}$

Where, $\mathrm{M}$ and $\mathrm{N}$ are size of an image.

A. Basic Evaluation: The Qualitative experimental evaluation for our method was performed on face dataset, that is FEI[24]

The implementation of iterative steering kernel regression worked on blemishes, Dark circles, pimples images on standard database which shown below.

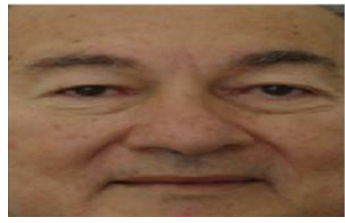

(a) Input Image

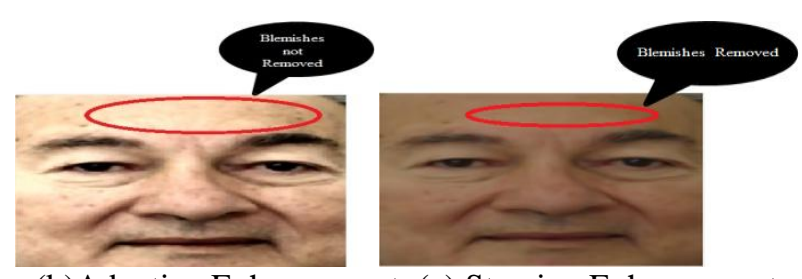

(b)Adaptive Enhancement (c) Steering Enhancement

Fig.9 Result for facial skin smoothening of Standard Database on Blemishes Image.

By doing number of several experiments in facial skin smoothing of standard database different parameters are used such as $\varepsilon, \lambda \& \alpha . \varepsilon$ is a small constant that prevents division by zero, $\lambda=1.95$ is used to balance the relative weights of the two terms, and $\alpha=0.98$ controls the propagation sensitivity of the gradient $(G)$ [5]. The

$260 * 360$ database image attains the 15750.1 MSE value and $32.925 \mathrm{~dB}$ PSNR value. In Iterative steering kernel regression for smoothening $\lambda=1$ and $\alpha=0.5$ is used for regularization at same dimension of image attains the 15777.8 MSE value and 32.902 dB PSNR value.

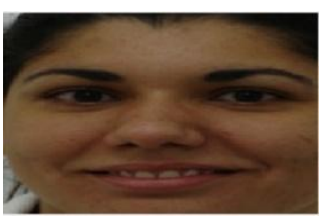

(a)Input Image

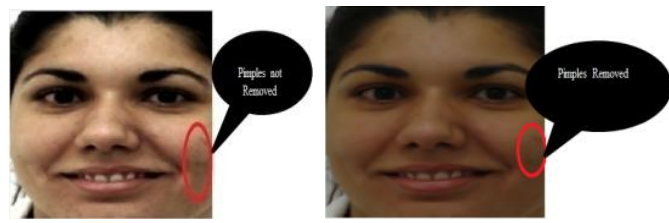

(b)Adaptive Enhancement (c) Steering Enhancement

Fig.10 Result for facial skin smoothening of Standard Database on pimple Image.

The $260 * 360$ dimensions of image attain the 7997.09 MSE value and $41.8431 \mathrm{db}$ PSNR value. In Iterative steering kernel regression for smoothening at same dimension of image attains the 8018.21 MSE value and $41.8036 \mathrm{~dB}$ PSNR value. The results are shown in above fig .10.

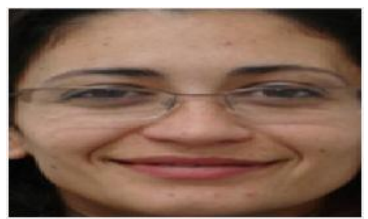

(a) Input Image

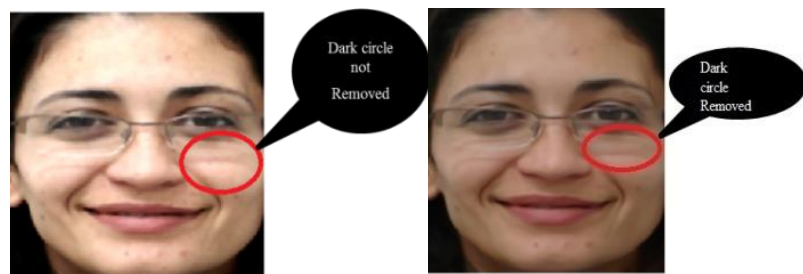

(b)Adaptive Enhancement

(c) Steering Enhancement

Fig.11 Result for facial skin smoothening of Standard Database on Dark circle Image.

The 260*360 dimensions of image attain the 7367.62 MSE value and $42.8082 \mathrm{db}$ PSNR value. In Iterative steering kernel regression for smoothening same dimension of image attains the 7379.85 MSE value and $42.7866 \mathrm{~dB}$ PSNR value. The results are shown in above fig. 11 .

B. The Qualitative experimental evaluation for our method was performed on General database.

The implementation of iterative steering kernel regression worked on blemishes, Dark circles, pimples images on General database which shown below. 
IJIREEICE

Vol. 5, Issue 1, January 2017

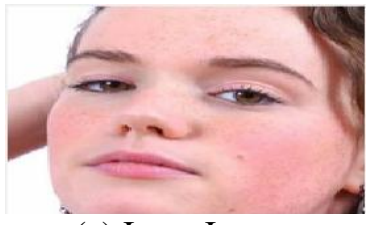

(a) Input Image

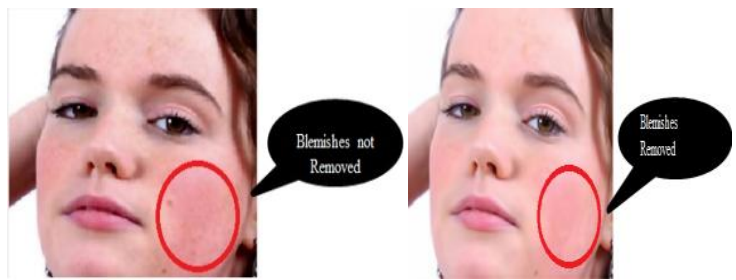

(b)Adaptive Enhancement(c) Steering Enhancement

Fig.12 Result for facial skin smoothening of General Database on Blemishes Image.

The $381 * 386$ dimensions of image attain the $1162.05 \mathrm{MSE}$ value and $66.8532 \mathrm{~dB}$ PSNR value. In Iterative steering kernel regression for smoothening same dimension of image attains the 11682.85 MSE value and $66.8444 \mathrm{~dB}$ PSNR value. The results are shown above in fig. 12 .

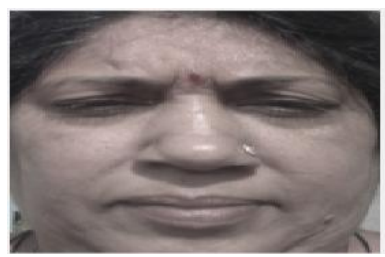

(a) Input Image

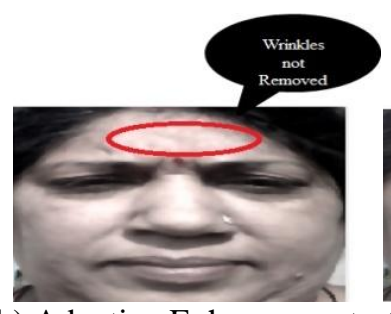

(b) Adaptive Enhancement

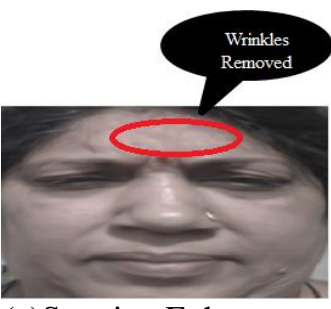

(c)Steering Enhancement
Fig.13 Result for facial skin smoothening of General Database on wrinkle Image.

The $1944 * 2592$ dimensions of image attain the 2854.97 MSE value and 55.1413 dB PSNR value. In Iterative steering kernel regression for smoothening at same dimension of image attains the 2860.14 MSE value and $55.1178 \mathrm{~dB}$ PSNR value. The results are shown above in fig. 13 .

Therefore the iterative steering kernel regression achieves better output than the adaptive region aware mask. It recovers much cleaner image and gives better MSE value as compare to traditional method. In traditional method only color of the image is changed which shows in above figure.
TABLE I Comparison of standard data base with Adaptive Region Aware Masks and Steering Kernel Regression.

\begin{tabular}{|c|c|c|c|c|}
\hline & \multicolumn{2}{|c|}{$\begin{array}{l}\text { Result of Facial Skin } \\
\text { Smoothening by } \\
\text { using Adaptive Regi } \\
\text {-on Aware Masks }\end{array}$} & \multicolumn{2}{|c|}{$\begin{array}{l}\text { Result Of Facial } \\
\text { Skin Smoothening } \\
\text { by using Steering } \\
\text { Kernel Regression }\end{array}$} \\
\hline Images & MSE & PSNR & MSE & PSNR \\
\hline 1 & 17505.5 & 31.55 & 17528 & 31.54 \\
\hline 2 & 12068.1 & 36.42 & 12089 & 36.40 \\
\hline 3 & 7997.09 & 41.843 & 8018.21 & 41.808 \\
\hline 4 & 17212.3 & 31.773 & 17232.1 & 31.7587 \\
\hline 5 & 13843.5 & 34.5953 & 13857.7 & 34.5819 \\
\hline 6 & 10925.1 & 37.6998 & 10940.7 & 37.6812 \\
\hline 7 & 4952.39 & 48.093 & 4964.54 & 48.061 \\
\hline 8 & 13110.8 & 35.324 & 13124.1 & 35.3108 \\
\hline 9 & 14353.5 & 34.1923 & 14371.9 & 34.1756 \\
\hline 10 & 7367.62 & 42.8082 & 7379.85 & 42.7866 \\
\hline 11 & 20449.3 & 29.5209 & 20470.7 & 29.5073 \\
\hline 12 & 15750.1 & 32.9251 & 15777.8 & 32.902 \\
\hline
\end{tabular}

The iterative steering kernel regression achieves better result as compare to the Adaptive region aware mask. There is a vast difference between the enhanced image using iterative steering kernel regression and the original image which is represented by large value of MSE.

TABLE II Comparison of General data base with adaptive Region Aware Masks and Steering Kernel Regression

\begin{tabular}{|c|c|c|c|c|}
\hline & \multicolumn{2}{|c|}{$\begin{array}{l}\text { Result of Facial Skin } \\
\text { Smoothening by } \\
\text { using Adaptive } \\
\text { Region Aware Masks }\end{array}$} & \multicolumn{2}{|c|}{$\begin{array}{l}\text { Result Of Facial } \\
\text { Skin Smoothening } \\
\text { by using Steering } \\
\text { Kernel Regression }\end{array}$} \\
\hline Images & MSE & PSNR & MSE & PSNR \\
\hline 1 & 5664.2 & 46.2237 & 5677.6 & 46.1927 \\
\hline 2 & 2854.97 & 55.1414 & 2860.14 & 55.1178 \\
\hline 3 & 1954.47 & 60.1556 & 1959.68 & 60.1205 \\
\hline 4 & 1785.94 & 61.288 & 1793.72 & 61.23 \\
\hline 5 & 7128.91 & 43.30 & 7132.89 & 43.29 \\
\hline 6 & 1085.83 & 67.7373 & 1089.46 & 67.6939 \\
\hline 7 & 5359.18 & 47.063 & 5366.09 & 47.0468 \\
\hline
\end{tabular}

The development of the final image of iterative steering kernel regression achieves better result as compare to the Adaptive region aware mask. There is a vast difference between the final image and the original image with respect to the MSE value. The MSE value is more it indicates our proposed method is more enhanced as compare to region aware mask method.

\section{CONCLUSION}

In this paper by using iterative steering kernel regression is implementation for facial skin smoothening based on edge preserving energy minimization method. Here the iterative steering kernel regression worked on two steps. Initially 


\section{International Journal of Innovative Research in Electrical, Electronics, Instrumentation and Control Engineering ISO 3297:2007 Certified}

Vol. 5, Issue 1, January 2017

the first step is estimated the gradient of input image. Next step is used to measure the main orientation of the local gradients in the image. In a next filtering stage, this orientation information is then used to adaptively "steer" the local kernel, resulting in elongated, elliptical contours spread along the directions of the local edge structure. The iterative steering kernel regression technique which worked on iterative steering algorithm as well as Q meter is used to identify the anisotropic patches and removes unwanted details such as wrinkles, spots, and blurriness on the image.

The visual results show that the proposed technique is highly effective for facial skin smoothening as compare to the traditional method in terms of lighting, smoothness, and color as well as by calculation with PSNR and MSE.

\section{REFERENCES}

[1] T. Leyvand, D. Cohen-Or, G. Dror, and D. Lischinski, "Data-driven enhancement of facial attractiveness," in Proc. ACM SIGGRAPH, 2008, pp.38:1-38:10

[2] K. Scherbaum, T. Ritschel, M. Hullin, T. Thormählen, V. Blanz, and H.-P. Seidel, "Computer-suggested facial makeup," Comput. GraphicsForum, vol.30, no. 2, pp. 485492, 20

[3] J. Fan, K. P. Chau, X. Wan, L. Zhai, and E. Lau, "Prediction of facial attractiveness from facial proportions," Patt. Recognit., vol. 45, no. 6,pp. 2326-2334, 2012.11.

[4] D. Lischinski, Z. Farbman, M. Uyttendaele, and R. Szeliski, "Interactive local adjustment of tonal values," ACM Trans. Graph., vol. 25, no. 3,pp. 646-653, 2006.

[5] T. Leyvand, D. Cohen-Or, G. Dror, and D. Lischinski, "Digital face beautification," in Proc. ACM SIGGRAPH Sketches, 2006, p. 169:1

[6] C.-W. Chen, D.-Y. Huang, and C.-S. Fuh, "Automatic skin color beautification," in Arts and Technology. Berlin/Heidelberg, Germany: SpringerVerlag, pp. 157-164, 2010

[7] M. P. Wand and M. C. Jones, Kernel Smoothing, ser. Monographs on Statistics and Applied Probability. New York: Chapman \& Hall, 1995.

[8] C. Florea, A. Capat " a, M. Ciuc, and P. Corcoran, "Facial enhancement "and beautification for HD video cameras," in Proc. IEEE Int. Conf. Consumer Electron., Jan. 2011, pp. 741-

[9] J. Canny, "A computational approach to edge detection," IEEE Trans.Patt. Anal. Mach. Intell., vol. 8, no. 6, pp. 679-698, Nov. 1986

[10] D. Guo and T. Sim, "Digital face makeup by example," in Proc. CVPR, 2009.

[11] G. B. Huang, M. Narayana, and E. Learned-Miller, "Towards unconstrained face recognition," in Proc. CVPR Workshop, 2008, pp. 1-8.

[12]. S. Mallat, A Theory for Multiresolution Signal Decomposition: the Wavelet Representation, IEEE Transactions on Pattern Analysis and Machine Intelligence 11 (7) (1989) 674-693.

[13] N. Wang, H. Ai, and F. Tang, "What are good parts for hair shape modeling," in Proc. CVPR, 2012, pp. 662-669.

[14] C. Scheffler, J. Odobez, and R. Marconi, "Joint adaptive colour modelling and skin, hair and clothing segmentation using coherent probabilistic index maps," in Proc. BMVC, 2011, pp. 53.1-53.11.

[15] A. Kae, K. Sohn, H. Lee, and E. Learned-Miller, "Augmenting CRFs with Boltzmann machine shape priors for image labeling," in Proc.CVPR, 2013, pp. 2019-2026.

[16] W.-S. Tong, C.-K. Tang, M. S. Brown, and Y.-Q. Xu, "Examplebased cosmetic transfer," in Proc. Pacific Conf. Comput. Graphics Applicat., 2007, pp. 211-218.

[17] V. Coetzee, S. J. Faerber, J. M. Greeff, C. E. Lefevre, D. E. Re, and D. I. Perrett, "African perceptions of female attractiveness," PLoS One, vol. 7, no. 10, p. e48116, 2012.
[18] I. D. Stephen, M. J. L. Smith, M. R. Stirrat, and D. I. Perrett, "Facial skin coloration affects perceived health of human faces," Int. J. Primatol.,vol. 30, no. 6, pp. 845-857, 2009.

[19] R. Russell, "Sex, beauty, and the relative luminance of facial features,"Perception, vol. 32, no. 9, pp. 1093-1108, 2003.

[20] P. Viola and M. J. Jones, "Robust real-time face detection," Int. J.Comput. Vision, vol. 57, no. 2, pp. 137-154, 2004.

[21] T. F. Cootes, C. J. Taylor, D. H. Cooper, and J. Graham, "Active shape models: Their training and application," Comput. Vision ImageUnderstand., vol. 61, no. 1, pp. 38-59, 1995.

[22] B. W. Silverman, "Spline smoothing: the equivalent variable kernel method," Ann. Statist., vol. 12, no. 3, pp. 898-916, Sep. 1984.

[23] X. Feng and P. Milanfar, "Multiscale principal components analysis for image local orientation estimation," presented at the 36th Asilomar Conf. Signals, Systems and Computers, Pacific Grove, CA, Nov. 2002.

[24]. FEI, Hillsboro, OR, USA. (2006). Face Database [Online]. Available: http://fei.edu.br/ cet/facedatabase.html.

\section{BIOGRAPHY}

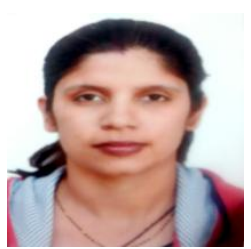

Priyanka Mehra received the B.E. degree in Instrumentation \& Control from IITM College RGPV, University Bhopal MP India, in 2007. Currently, she is a pursuing Master of Engineering from Imperial college of Engineering and Research, Wagholi, Pune, Maharashtra SPPU University. Her current research interests include," Facial Skin Smoothening by Iterative Steering Kernel Regression. 